\title{
Associação entre o ângulo de Norberg, o percentual de cobertura da cabeça femoral, o índice cortical e o ângulo de inclinação em cães com displasia coxofemoral
}

\author{
[Associations among Norberg angle, percentage of femoral head coverage, \\ cortico-medullary index, and femoral inclination angle in dogs with hip dysplasia] \\ G.L.T. Vieira, R.C.S. Tôrres*, G.S. Barros, B.D. Rocha, C.M.F. Rezende \\ Escola de Veterinária - UFMG \\ Av. Antônio Carlos, 6627 \\ 30123-970 - Belo Horizonte, MG
}

\begin{abstract}
RESUMO
Foram avaliadas 386 radiografias da articulação coxofemoral, sendo 220 de cães da raça Pastor Alemão, 112 machos e 108 fêmeas, e 166 da raça Labrador Retriever, 69 machos e 97 fêmeas. As radiografias foram classificadas segundo o grau de displasia coxofemoral (DCF), e foram mensurados o ângulo de inclinação, o ângulo de Norberg, o índice cortical e o percentual de cobertura da cabeça femoral de ambos os lados. As variáveis foram associadas mediante análise estatística multivariada de componentes principais. As variáveis índice cortical e ângulo de inclinação foram inversamente associadas. A raça Pastor Alemão apresentou valores de índice cortical e graus de DCF mais baixos em relação à raça Labrador Retriever. Maior ângulo de inclinação foi associado a menor ângulo de Norberg e menor percentual de cobertura. Animais mais velhos apresentaram menor ângulo de Norberg, menor porcentagem de cobertura e maior grau de DCF. Nas fêmeas, foram observados menor porcentagem de cobertura, menor ângulo de Norberg e maior grau de DCF. Pode-se concluir que o ângulo de inclinação e o índice cortical não demonstraram associação com a DCF.
\end{abstract}

Palavras-chave: cão, displasia coxofemoral, ângulo de Norberg, índice cortical, ângulo de inclinação, percentual de cobertura

\begin{abstract}
A total of 386 radiographs of the pelvis were evaluated, being 220 of German Shepherd dogs (112 males and 108 females) and 166 of Labrador Retrievers (69 males and 97 females). The radiographs were degree classified regarding the of hip dysplasia (DHD). The Norberg and inclination angles, the corticomedullary index, and the percentage coverage of the femoral head were measured and associated using multivariate statistical technique (principal component analysis). The cortico-medullary index and the inclination angle were inversely associated. The results indicated that German Shepherd Dogs showed lower cortico-medullary index and DHD compared with Labrador Retrievers. The higher the inclination angle, the lower the Norberg angle and percentage coverage of the femoral head. It was observed that with age, animals showed lower Norberg angles and percentage coverage of the femoral head, thus more hip dysplasia severity. Females were associated to lower femoral head coverage and Norberg angles, and more severe DHD. Femoral inclination angle and cortico-medullary index did not demonstrate a correlation to $D H D$.
\end{abstract}

Keywords: hip dysplasia, angle of Norberg, cortical index, inclination angle, percentile of covering

Recebido em 30 de dezembro de 2009

Aceito em 10 de agosto de 2010

*Autor para correspondência (corresponding author)

E-mail: rtorres@vet.ufmg.br

Apoio: FAPEMIG 


\section{INTRODUÇÃO}

A displasia coxofemoral (DCF) é uma doença genética de alta prevalência, debilitante, que causa dor, desconforto e diminuição da vida útil, em cães. Apesar de inúmeras pesquisas e dos bem-intencionados programas de acasalamento a doença continua a exercer um grande impacto financeiro e emocional em proprietários e criadores (Fries e Remedios, 1995). Todas as raças de cães podem ser acometidas, porém a doença é mais comum em raças de porte grande. A prevalência em algumas raças é acima de $70 \%$, sendo a doença ortopédica mais comum dos cães (Tôrres, 1993).

A DCF tem sido intensivamente estudada desde a sua primeira descrição em cães, em 1935 (Banfield et al., 1996). Os cães geneticamente predispostos à DCF nascem com articulações coxofemorais normais. As anormalidades associadas à patologia se desenvolvem à medida que os filhotes crescem (Tomlinson e McLaughlin Jr., 1996). A DCF é de origem multifatorial, sendo que fatores como idade, raça, sexo, tamanho do animal, conformação, nutrição e massa muscular pélvica têm sido implicados no desenvolvimento da doença, entretanto a frouxidão articular é considerada o fator primário (Wallace, 1987; Martinez, 1997; Tôrres e al., 2005).

Os objetivos deste trabalho foram avaliar a associação de fatores como ângulo de Norberg, percentual de cobertura da cabeça femoral, ângulo de inclinação, índice cortical, raça, sexo e idade e verificar quais variáveis relacionam-se entre si e com a DCF, em duas raças de cães com alta predisposição genética à doença.

\section{MATERIAL E MÉTODOS}

Foram avaliadas 386 radiografias das articulações coxofemorais em cães de ambos os sexos, sendo 220 da raça Pastor Alemão - 112 machos e 108 fêmeas - e 166 da raça Labrador Retriever - 69 machos e 97 fêmeas -, com idades entre sete meses e oito anos; 97 eram cães normais e 289, displásicos. Os animais, provenientes de criatórios particulares e do canil da Polícia Militar do Estado de Minas Gerais, foram encaminhados para a realização de laudo radiográfico de DCF entre abril de 1994 e julho de 2006.
Para a realização dos exames radiográficos, todos os animais foram sedados utilizando-se cloridrato de xilazina (Rompum R - Lab. Bayer do Brasil S. A.) (1 mg/kg - IM). Foi realizada radiografia na posição padrão utilizada para o diagnóstico da DCF, na qual os animais são posicionados em decúbito dorsal, com os membros posteriores completamente estendidos, os fêmures paralelos entre si e em relação à coluna vertebral e a pelve em simetria. Utilizaram-se aparelho de Raio-X (Aparelho VMI - VMI Ind. Aparelhos Radiológicos ) e filmes (Kodak MXG/Plus Filme para Raio-X Médico - Kodak Brasileira Com. Ind. Ltda.) de tamanho $30 \times 40 \mathrm{~cm}$.

As radiografias foram avaliadas segundo a classificação proposta pela FCI (Brass et al., 1978) e adotada pelo serviço de radiologia do Hospital Veterinário da Escola de Veterinária da UFMG. Os animais foram classificados como normal, suspeito de DCF, DCF leve, DCF média e DCF grave. Em todas as radiografias, foram mensurados em ambos os lados o ângulo de Norberg, o percentual de cobertura da cabeça femoral, o ângulo de inclinação e o índice cortical. A mensuração do ângulo de Norberg (Fig. 1) foi realizada segundo Brass et al. (1978). $O$ percentual de cobertura (PC) da cabeça femoral (Fig. 1) foi calculado segundo a fórmula: $\mathrm{PC}=(\mathrm{a} / \mathrm{b}) \times 100$, em que $\mathrm{a}=$ comprimento da cabeça femoral posicionada dentro do acetábulo $\mathrm{e} b=$ diâmetro da cabeça do fêmur (Tomlinson e Johnson, 2000). O ângulo de inclinação (AI) (Fig. 2) foi mensurado conforme descrito por Hauptman et al. (1979), e o índice cortical (IC) (Fig. 2) foi calculado conforme a fórmula: $\mathrm{IC}=$ $(\mathrm{AB}-\mathrm{CD}) / \mathrm{AB}$, em que $\mathrm{AB}=$ espessura total do osso e $C D=$ espessura da medular óssea (Hartung e Hasselt, 1988).

As associações entre as variáveis (grau de DCF, raça, sexo, idade, ângulo de Norberg, percentual de cobertura da cabeça femoral, índice cortical e ângulo de inclinação) foram avaliadas segundo uma análise estatística multivariada de componentes principais, utilizando-se o programa InfoStat da Universidade de Córdoba/Argentina. A representação gráfica dessas variáveis no sistema tridimensional permitiu verificar em um sistema de coordenadas as associações existentes entre elas. 


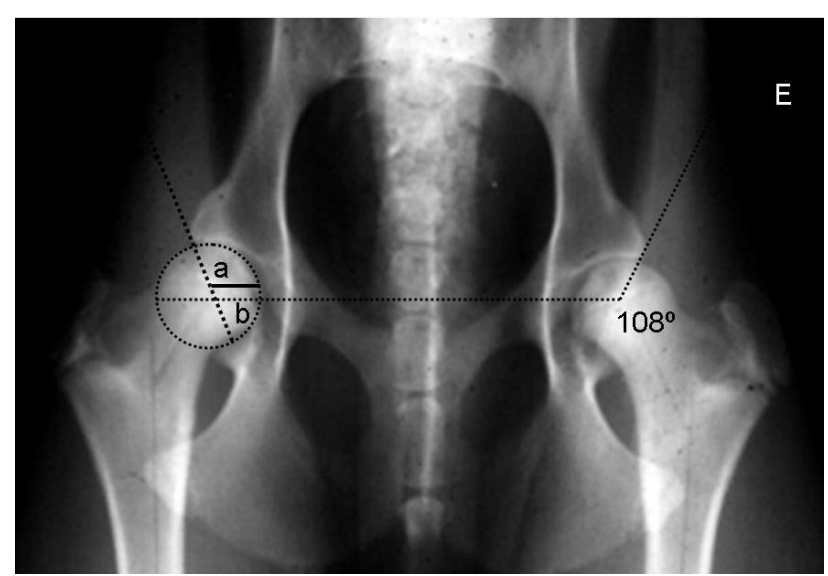

Figura 1. Imagem radiográfica da articulação coxofemoral de cão. No fêmur direito está ilustrado como o percentual de cobertura da cabeça femoral (PC) é calculado, e no esquerdo está ilustrada a mensuração do ângulo de Norberg.

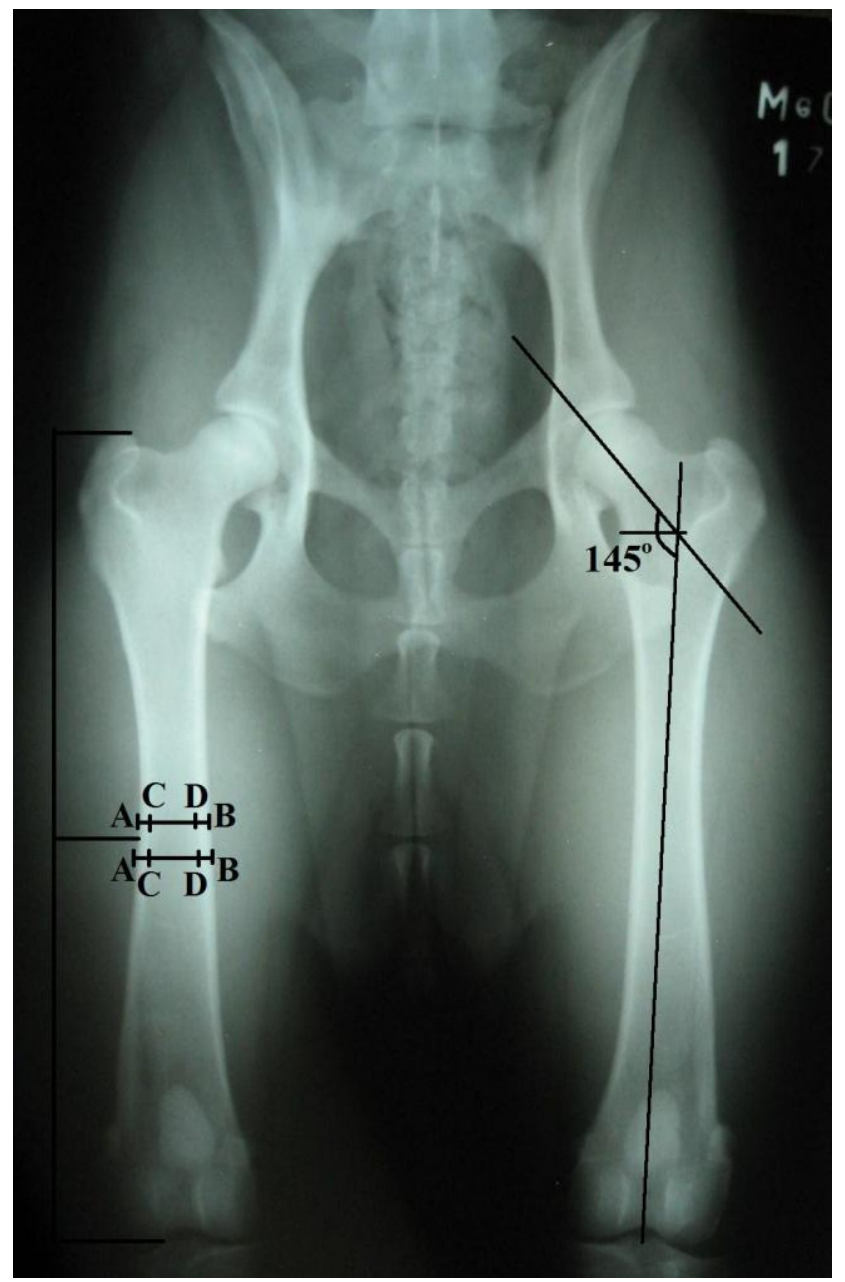

Figura 2. Imagem radiográfica da articulação coxofemoral de cão na posição padrão para o diagnóstico da displasia coxofemoral. No fêmur direito está ilustrado o cálculo do índice cortical (IC), e no esquerdo a mensuração do ângulo de inclinação (AI) $\left(145^{\circ}\right)$. 


\section{RESULTADOS E DISCUSSÃO}

Neste estudo, a primeira, a segunda e a terceira componente principal explicaram cerca de $34 \%$, $22 \%$, e $13 \%$ da variação dos dados, respectivamente, totalizando $69 \%$ da variação.
Foram utilizadas as três primeiras componentes principais para a elaboração da figura. A Fig. 3 ilustra as projeções das variáveis nos planos traçados pelo primeiro, segundo e terceiro eixos fatoriais, demonstrando as correlações encontradas entre as variáveis.

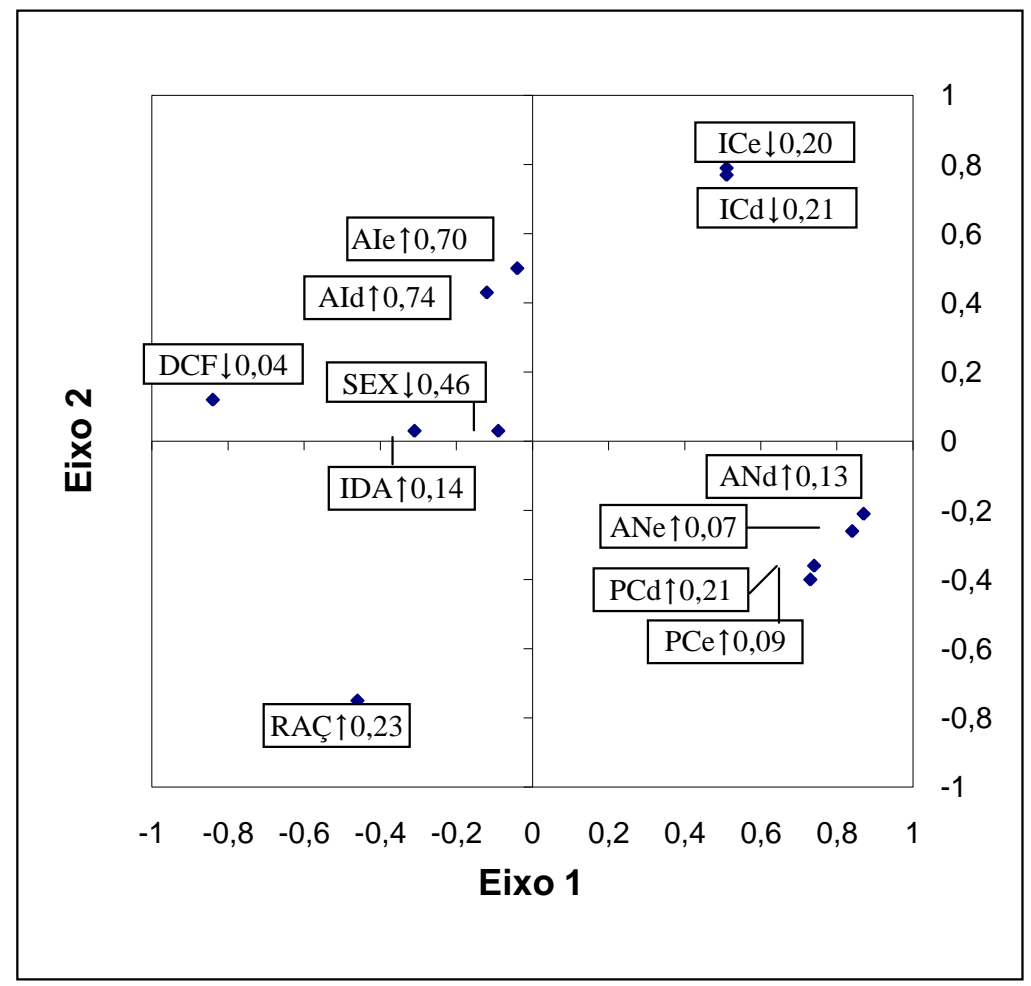

Figura 3. Representação gráfica das variáveis estudadas no sistema tridimensional originado pelos três primeiros eixos (componentes) principais (direção e coordenadas do terceiro eixo representadas pela direção das setas). Variáveis: raça (RAÇ), idade (Ida), sexo (SEX), grau de DCF (DCF), ângulo de Norberg direito (ANd), ângulo de Norberg esquerdo (ANe), percentual de cobertura direito (PCd), percentual de cobertura esquerdo (PCe), ângulo de inclinação direito (AId), ângulo de inclinação esquerdo (AIe), índice cortical direito (ICd), índice cortical esquerdo (Ice).

As variáveis ângulo de inclinação direito e ângulo de inclinação esquerdo são diretamente proporcionais e com forte associação positiva entre elas, sendo o mesmo fato observado entre o índice cortical dos fêmures direito e esquerdo. Isso sugere que as bilateralidades estão altamente associadas, semelhante aos relatos de Ocal et al. (2004) e Palierne et al. (2006), que observaram não haver diferenças significativas entre a morfologia dos fêmures direito e esquerdo. Markel e Sielman (1993) afirmaram que essa simetria bilateral entre os fêmures direito e esquerdo é importante para a utilização do membro contralateral como controle em estudos ortopédicos.
Forte associação positiva foi também observada entre o ângulo de Norberg direito e o esquerdo e a porcentagem de cobertura das cabeças femorais direita e esquerda, tanto em animais displásicos como normais. Além da similaridade na morfologia óssea dos fêmures direito e esquerdo, espera-se encontrar essa associação também em animais displásicos, já que a DCF geralmente é bilateral (McLaughlin Jr. e Tomlinson, 1996). Desse modo, esperam-se intensidades iguais ou similares de frouxidão articular e artrose nas articulações coxofemorais direita e esquerda (Cook et al., 1996; Citi et al., 2005), sendo a amostra deste estudo predominantemente de animais com apresentação bilateral da patologia. 
Neste estudo, foi evidenciado que as variáveis raça e índices corticais (direito e esquerdo) foram inversamente proporcionais. A raça Pastor Alemão apresentou menor valor de índice cortical. A espessura da cortical óssea está associada à rigidez do osso, sendo essa rigidez dependente da quantidade de forças a que esse osso está submetido (Palierne et al., 2006). As forças de reação da articulação coxofemoral em cães variam de 150 a $165 \%$ do peso corporal durante a deambulação (Arnoczky e Torzilli, 1981; Page et al., 1993). Como o peso corporal médio de cães adultos da raça Labrador Retriever é, em média, $2,4 \pm 0,3 \mathrm{~kg}$ maior que o da raça Pastor Alemão (Helmink et al., 2000), é esperado que os cães Labrador Retriever possuam índice cortical maior que os Pastores Alemães, que são mais leves e, portanto, a carga de forças que incidem sobre o fêmur é menor. Essa diferença morfológica é devido tanto à predeterminação genética da raça quanto à adaptação biológica do osso à carga de forças.

Foi demonstrado que, nos casos das doenças esqueléticas, a relação entre osso compacto e osso esponjoso pode se alterar, ocorrendo variação no volume ósseo e na concentração de cálcio no tecido ósseo (Hartung e Hasselt, 1988). Porém, neste estudo, não foi observada associação entre o índice cortical e a DCF, semelhante aos relatos de Hartung e Hasselt (1988) e Tôrres (1993). Os valores de média do índice cortical de cães hígidos foram $0,2731 \pm 0,0325$ e $0,3628 \pm 0,0326$, e de cães displásicos foram $0,2735 \pm 0,0303 \mathrm{e}$ 0,3558 $\pm 0,0367$ nas raças Pastor Alemão e Labrador Retriever, respectivamente (Tab. 1).

Tabela 1. Valores médios do ângulo de Norberg, percentual de cobertura da cabeça femoral, ângulo de inclinação e índice cortical em 386 cães hígidos e displásicos das raças Pastor Alemão e Labrador Retriever

\begin{tabular}{ccccc}
\hline $\begin{array}{c}\text { Variável/ } \\
\text { Diagnóstico } \\
\text { radiográfico }\end{array}$ & \multicolumn{2}{c}{ Pastor Alemão } & \multicolumn{2}{c}{ Labrador Retriever } \\
\cline { 2 - 5 }$(\mathrm{n}=36)$ & $106 \pm 3,44$ & $\begin{array}{c}\text { Displásico } \\
(\mathrm{n}=184)\end{array}$ & $\begin{array}{c}\text { Hígido } \\
(\mathrm{n}=61)\end{array}$ & $\begin{array}{c}\text { Displásico } \\
(\mathrm{n}=105)\end{array}$ \\
\hline AN $\left(^{\circ}\right)$ & $53,42 \pm 10,09$ & $106,0 \pm 4,03$ & $100,3 \pm 7,66$ \\
$\mathrm{PC}(\%)$ & $140,0 \pm 4,62$ & $43,43 \pm 11,37$ & $50,23 \pm 5,11$ & $42,26 \pm 9,03$ \\
$\mathrm{AI}\left(^{\circ}\right)$ & $143,1 \pm 6,93$ & $144,7 \pm 5,99$ & $144,5 \pm 6,43$ \\
IC & $0,2731 \pm 0,0325$ & $0,2735 \pm 0,0303$ & $0,3628 \pm 0,0326$ & $0,3558 \pm 0,0367$
\end{tabular}

AN: ângulo de Norberg; PC: percentual de cobertura da cabeça femoral; AI: ângulo de inclinação; IC: índice cortical.

O índice cortical foi inversamente associado ao ângulo de inclinação neste estudo. A estimulação contínua do osso com uma carga de forças diferente do padrão fisiológico induz uma resposta remodeladora para otimizar o esqueleto para a nova condição. No fêmur normal, a cortical medial está principalmente sob o efeito de forças de compressão, enquanto a lateral está sob o efeito das forças de tração (Page et al., 1993). As forças de compressão promovem deposição e produção de osso, resultando no aumento da espessura cortical, enquanto as forças de tração estimulam a reabsorção e consequentemente diminuição da espessura cortical (Pernell et al., 1994). A resposta do osso às sobrecargas mecânicas é imediata e envolve reações celulares e teciduais. Os osteoblastos e osteócitos reagem em resposta às alterações na tensão óssea, refletindo uma adaptação à sobrecarga imposta pelo ambiente (Sommerfeldt e Rubin, 2001). O aumento do ângulo de inclinação altera o ângulo da força total sobre a articulação coxofemoral, modificando o seu valor normal de $69^{\circ}$ para até $80^{\circ}$ (Weigel e Wasserman, 1992). O ângulo da força total sobre a articulação exerce grande influência na distribuição das forças sobre o fêmur, podendo ocasionar alterações na espessura cortical óssea (Taylor et al., 1996).

A média dos ângulos de inclinação das raças Pastor Alemão e Labrador Retriever nos animais considerados normais foi de $140,0 \pm 4,62^{\circ} \mathrm{e}$ $144,7 \pm 5,99{ }^{\circ}$ e nos displásicos $143,1 \pm 6,93^{\circ}$ e $144,5 \pm 6,43^{\circ}$, respectivamente (Tab. 1). Observou-se que maior ângulo de inclinação estava associado a menor ângulo de Norberg e a menor percentual de cobertura da cabeça do fêmur pelo acetábulo. Apesar de a subluxação da cabeça femoral representar um fator de risco 
significativo para o desenvolvimento de doença articular degenerativa (DAD) da articulação coxofemoral (Kealy et al., 1992), o ângulo de inclinação não esteve associado à DCF, semelhante a estudos anteriores (Hauptman et al., 1985; Tôrres, 1993; Banfield et al., 1996; Sarierler, 2004).

Foi observado que o grau de DCF estava positivamente correlacionado com a idade e variou inversamente com o ângulo de Norberg e com a porcentagem de cobertura da cabeça do fêmur. Uma das alterações radiográficas da DCF é a mudança no formato do acetábulo, caracterizada pelo arrasamento das bordas dorsal e crânio-lateral. O acetábulo raso vai contribuir potencialmente para um menor percentual de cobertura. Quanto menos saliente for a proeminência cranial do acetábulo, menor será o ângulo de Norberg (Tomlinson e Johnson, 2000), sendo essa associação positiva entre o ângulo de Norberg e o percentual de cobertura, confirmada neste e em outros estudos (Lust et al., 1993; Tomlinson e Johnson, 2000). Como a gravidade da DCF aumenta com o avanço da idade (Lust et al., 1993), é esperado que à medida que o animal envelheça, ocorra a progressão da doença e o agravamento da DAD, com maior arrasamento do acetábulo, resultando em menor ângulo de Norberg, menor porcentagem de cobertura e maior grau de DCF. Esse resultado indica que, devido ao efeito da idade, todos os animais devem ser avaliados aproximadamente em faixa etária semelhante.

Em relação à associação da raça com a DCF, neste estudo a raça Pastor Alemão foi associada ao menor grau de DCF em comparação à raça Labrador Retriever. Alguns autores citaram que a susceptibilidade ao desenvolvimento de DCF varia com as raças. Smith et al. (1995) analisaram os fatores de risco para a DCF entre um grupo de cães da raça Pastor Alemão e um grupo de cães de raças variadas e observaram que os Pastores Alemães têm um risco seis vezes maior de desenvolverem osteoartrose da articulação coxofemoral que os cães do segundo grupo.

Neste estudo, as fêmeas estavam associadas à menor porcentagem de cobertura, menor ângulo de Norberg e maior grau de DCF. Resultado semelhante foi encontrado em estudo anterior (Swenson et al., 1997). Porém, diferenças na susceptibilidade à DCF em relação ao sexo não foram encontradas em outros estudos (Maki et al., 2000; Rettennmaier at al., 2002). Segundo Swenson et al. (1997), essa diferença na prevalência da DCF em relação ao sexo pode ser explicada por um efeito direto dos genes localizados nos cromossomos sexuais, ou por um efeito nas características sexuais secundárias codificadas pelos mesmos genes (diferenças na taxa de crescimento, hormônios sexuais, ou padrões comportamentais). Para a DCF, na qual a expressão da doença depende de vários genes, a última explicação é a mais provável.

\section{CONCLUSÕES}

As variáveis que mais se associaram à DCF foram o ângulo de Norberg, o percentual de cobertura da cabeça femoral, a idade, a raça e o sexo. O ângulo de inclinação e o índice cortical não demonstraram associação com a DCF.

\section{AGRADECIMENTOS}

Ao Professor Ivan Sampaio, pela ajuda com a análise estatística deste trabalho.

\section{REFERÊNCIAS BIBLIOGRÁFICAS}

ARNOCZKY, S.P.; TORZILLI, P.A. Biomechanical analysis of forces acting about the canine hip. Am. J. Vet. Res., v.42, p.15811585, 1981.

BANFIELD, C.M.; BARTELS, J.E.; HUDSON, J.A. et al. A retrospective study of canine hip dysplasia in 116 military working dogs. Part I: Angle measurements and Orthopedic Foundation for Animals (OFA) grading. J. Am. Vet. Med. Assoc., v.32, p.413-422, 1996.

BRASS, W.; FREUDIGER, U.; MULLER, L.F. et al. Bericht der huftgelenkdysplasie-komission. Kleintier Praxis, v.23, p.169-180, 1978.

CITI, S.; VIGNOLI, M.; MODENATO, M. et al. A radiological study of the incidence of unilateral canine hip dysplasia. Schweiz. Archiv Tierheilkund., v.147, p.173-178, 2005.

COOK, J.L.; TOMLINSON, J.L.; CONSTANTINESCU, G.M. Pathophysiology, diagnosis, and treatment of canine hip dysplasia. Comp. Cont. Educ. Pract. Vet., v.18, p.853-867, 1996. 
FRIES, C.L.; REMEDIOS, A.M. The pathogenesis and diagnosis of canine hip dysplasia: A review. Can. Vet. J., v.36, p.494501, 1995.

HARTUNG, K.; HASSELT, S.C. Morphometrische untersuchungen am femurknochen des hundes. Berl. Munch. Tierarztl. Wochenschr., v.101, p.15-79, 1988.

HAUPTMAN, J.; CARDINET, G.H.; MORGAN, J.P. et al. Angles of inclination and anteversion in hip dysplasia in the dog. Am. J. Vet. Res., v.46, p.2033-2036, 1985.

HAUPTMAN, J.; PRIEUR, W.D.; BUTLER, H.C. et al. The angle of inclination of the canine femoral head and neck. Vet. Surg., v.8, p.74-77, 1979.

HELMINK, S.K.; SHANKS, R.D.; LEIGHTON, E.A. Breed and sex differences in growth curves for two breeds of dog guides. J. Anim. Sci., v.78, p.27-32, 2000.

KEALY, R.D.; OLSSON, S.E.; MONTI, K.L. et al. Effects of limited food consumption on the incidence of hip dysplasia in growing dogs. $J$. Am. Vet. Med. Assoc., v.201, p.857-863, 1992.

LUST, G.; WILLIAMS, A.J.; BURTONWURSTER, N. et al. Joint laxity and its association with hip dysplasia in Labrador Retrievers. Am. J. Vet. Res., v.54, p.1990-1999, 1993.

MÄKI， K.; LIINAMO, A.E.; OJALA， M. Estimates of genetic parameters for hip and elbow dysplasis in Finnish Rottweilers. J. Anim. Sci., v.78, p.1141-1148, 2000.

MARKEL, M.D.; SIELMAN, E. Radiographic study of homotypic variation of long bones in dogs. Am. J. Vet. Res., v.54, p.2000-2003, 1993.

MARTINEZ, S.A. Congenital conditions that lead to osteoarthritis in the dog. Vet. Clin. N. Am.: Small Anim. Pract., v.27, p.735-758, 1997.

McLAUGHLIN Jr., R.; TOMLINSON, J. Radiographic diagnosis of canine hip dysplasia. Vet. Med., v.91, p.36-47, 1996.
OCAL, M.K.; KARA, M.E.; TURAN, E. Computed tomographic measurements of the hip morphology of 10 healthy German shepherd dogs. Vet. Rec., v.155, p.392-395, 2004.

PAGE, A.E.; ALLAN, C.; JASTY, M. et al. Determination of loading parameters in the canine hip in vivo. J. Biomec., v.26, p.571-579, 1993.

PALIERNE, S.; ASIMUS, E.; MATHON, D. et al. Geometric analysis of the proximal femur in a diverse sample of dogs. Res. Vet. Sci., v.80, p.243-252, 2006.

PERNELL, R.T.; GROSS, R.S.; MILTON, J.L. et al. Femoral strain distribution and subsidence after physiological loading of a cementless canine femoral prosthesis : the effects of implant orientation, canal fill, and implant fit. Vet. Surg., v.23, p.503-518, 1994.

RETTENNMAIER， J.L.; KELLER， G.G.; LATTIMER, J.C. et al. Prevalence of canine hip dysplasia in a veterinary teaching hospital population. Vet. Rad. Ultrasound, v.43, p.313318, 2002.

SARIERLER, M. Comparison of femoral inclination angle measurements in dysplasic and nondysplasic dogs of different breeds. Ac. Vet. Hung., v.52, p.245-252, 2004.

SMITH, G.K.; POPOVITCH, C.A.; GREGOR, T.P. et al. Evaluation of risk factors for degenerative joint disease associated with hip dysplasia in dogs. J. Am. Vet. Med. Assoc., v.206, p.642-647, 1995.

SOMMERFELDT, D.W.; RUBIN, C.T. Biology of bone and how it orchestrates the form and function of the skeleton. Eur. Spine J., v.10, p.S86-S85, 2001

SWENSON, L.; AUDELL, L.; HEDHAMMAR, A. Prevalence and inheritance of and selection for hip dysplasia in seven breeds of dogs in Sweden and benefit: cost analysis of a screening and control program. J. Am. Vet. Med. Assoc., v.210, p.207-214, 1997.

TAYLOR, M.E.; TANNER, K.E.; FREEMAN, M.A.R. et al. Stress and strain distribution within the intact femur: compression or bending? Med. Engin. Phys., v.18, p.122-131, 1996. 
TOMLINSON, J.L.; JOHNSON, J.C. Quantification of measurement of femoral head coverage and Norberg angle within and among four breeds of dogs. Am. J. Vet. Res., v.61, p.1492-1500, 2000.

TOMLINSON, J.; McLAUGHLIN Jr., R. Canine hip dysplasia: developmental factors, clinical signs, and initial examination steps. Vet. Med., v.91, p.26-33, 1996.

TÔRRES, R.C.S. Prevalência da displasia coxofemoral em cães da raça Pastor Alemão, 1993. 69f. Dissertação (Mestrado) - Escola de Veterinária, Universidade Federal de Minas Gerais, Belo Horizonte.
TÔRRES, R.C.S.; ARAÚJO, R.B.; REZENDE, C.M.F. Distrator articular no diagnóstico radiográfico precoce da displasia coxofemoral em cães. Arq. Bras. Med. Vet. Zootec., v.57, p.27-34, 2005.

WALLACE, L.J. Canine hip dysplasia: past and present. Sem. Vet. Med. and Surg. (Small Anim.), v.2, p.92-106, 1987.

WEIGEL, J.P.; WASSERMAN, J.F. Biomechanics of the normal and abnormal hip joint. Vet. Clin. N. Am.: Small Anim. Pract., v.22, p.513-528, 1992. 